\title{
Condições higiênico-sanitárias de Unidades de Alimentação e Nutrição Hospitalares da Região Oeste de Santa Catarina
}

\author{
Maiara Techio Pederssetti ${ }^{1}$ e Tiffany Prokopp Hautrive ${ }^{2}$
}

As Unidades de Alimentação e Nutrição Hospitalares (UANs) devem oferecer uma alimentação adequada, balanceada e segura. A alimentação desta clientela integra tratamento clínico e deve contribuir para a recuperação dos pacientes. O objetivo deste trabalho foi avaliar as condições higiênico-sanitárias de quatro UANs hospitalares da região oeste do estado de Santa Catarina (SC). Utilizou-se uma lista de verificação baseada nas resoluções RDC 216/04 e RDC 275/02 da vigilância sanitária avaliando 11 itens em relação às Boas Práticas de Fabricação (BPF) e aos Procedimentos Operacionais Padronizados (POPs). Os resultados obtidos mostraram algumas irregularidades nas UANs, principalmente naquelas que que não mantinham nutricionista no local. Apesar das irregularidades apresentadas, nenhuma UAN foi classificada no Grupo 3, considerado o de pior qualidade higiênico sanitária. As UANs A e B foram classificadas no Grupo 2 - Regular, e as UANs C e D foram classificadas no Grupo 1 - Bom. Apesar das quatro UANs apresentarem um bom percentual de adequação e nenhuma encontrarse na classificação Ruim, as UANs que se encaixaram no Grupo 2 merecem uma atenção especial quanto à qualidade higiênico sanitária, pois não possuem um profissional responsável no local aumentando o risco de contaminação alimentar durante o preparo de dietas hospitalares.

Palavras-chave: Serviços de alimentação, Vigilância Sanitária, higiene, Segurança Alimentar.

\section{Sanitary-hygienic conditions of Food and Nutrition Hospital Units in the West Region of Santa Catarina}

Hospital food services should provide adequate, balanced, and safe nutrition, once it is part of clinical treatment and should contribute to the patients' recovery. The aim of this study was to evaluate the sanitary conditions of four hospital food services in the west region of SC state. A checklist based on the resolutions RDC 216/04 and RDC 275/02 was used, with 11 items assessing health surveillance in relation to Good Manufacturing Practices (GMP) and Standard Operating Procedures (SOPs). The results showed some irregularities in hospital food services, especially those without the presence of a nutritionist. Despite the irregularities, no UAN was classified in Group 3 - considered the worst sanitary quality. The food services A and B were classified in Group 2 - Regular, and the food services C and D were classified in Group 1 - Good. Despite the four hospital food services presented a good percentage of adequacy, and none was classified as Poor, the food services of Group 2 need special attention

1 Nutricionista e Especialista em Nutrição Humana com ênfase em Nutrição Coletiva (UAN) - Universidade Região de Chapecó - UNOCHAPECÓ. E-mail: tecchio@unochapeco.edu.br

2 Doutora em Ciência e Tecnologia de Alimentos pela Universidade Federal de Santa Catarina. Nutricionista responsável pelo Serviço de Alimentação Hospitalar Clínica do Hospital de Guarnição de Santa Maria - RS. Endereço para correspondência: Rua Benjamim Constant no 930, apto. 116. CEP 97050-020. Santa Maria - RS. E-mail: tiffanyhautrive@yahoo.com.br 
about sanitary quality because they are not overseen by a professional nutritionist in loco, increasing the risk of contamination during food preparation in the hospitals.

Keywords: Food service, Health and Sanitation, hygiene, Food Safety.

\section{INTRODUÇÃO}

As doenças transmitidas por alimentos (DTA's) se destacam como um dos fatores que mais contribuem para a morbidade nos países da América latina e um dos maiores problemas de saúde pública no mundo contemporâneo ${ }^{[1]}$.

Grande parte dos problemas que atingem parcelas significativas da população mundial está relacionada com questões alimentares e nutricionais, seja pela ausência do alimento, pela má qualidade da alimentação ou por condições de vida e saúde que impedem o aproveitamento adequado do alimento disponível [2].

De acordo com estudos estatísticos da Organização Mundial de Saúde (OMS), mais de 60\% dos casos de doenças de origem alimentar decorrem do descuido higiênico-sanitário de manipuladores, das técnicas inadequadas de processamento e da deficiência de higiene da estrutura física, utensilios e equipamentos [3].

Para evitar as doenças de origem alimentar os alimentos devem ser preparados de modo a garantir a segurança do consumidor, adotando medidas de prevenção e controle em todas as etapas da cadeia produtiva [4]. Nesse sentido, o controle higiênicosanitário dos alimentos constitui fator preponderante para prevenção das DTA's [5].

Para Schneider ${ }^{[6]}$ as Unidades de Alimentação e Nutrição (UANs) constituem importantes setores dentro das empresas onde estão estabelecidas. Mais ainda, em se tratando de hospitais, onde o fornecimento de alimentos destina-se ao preparo de dietas especializadas que visam à recuperação e tratamento dos pacientes. Dentre os objetivos das UANs em uma organização hospitalar, verifica-se como prioridade o fornecimento de alimentação adequada, balanceada e segura.
Ainda para Martinelli [7] em uma unidade hospitalar, a alimentação e a nutrição têm como principal finalidade restaurar a saúde dos pacientes, servindo como um importante fator coadjuvante ao tratamento médico, e ajudando a oferecer o aporte necessário de nutrientes.

Souza e Proença ${ }^{[8]}$ destacam que as refeições hospitalares além da qualidade nutricional e sanitária, devem agregar também características psicossensoriais e simbólicas aos pacientes, pois a alimentação para o ser humano envolve aspectos além do atendimento às necessidades fisiológicas, como também psicológicas, sociais e econômicas.

Sousa et al. ${ }^{[9]}$ referem que apesar de haver um avanço tecnológico sobre as técnicas de conservação e higiene dos alimentos, as DTA's são reconhecidas como um grave problema de saúde pública devido os manipuladores de alimentos que, em sua maioria, não detêm conhecimentos adequados higiênicos, o que se reflete no seu comportamento durante a higiene pessoal e de equipamentos e utensílios.

Existem normas e diretrizes que preconizam a adoção das Boas Práticas de fabricação em todos os estabelecimentos de produção e comercialização de alimentos e afins, com o intuito de reduzir a incidência dos surtos alimentares e garantir a saúde da população [10]. As Boas Práticas compreendem o controle de matérias-primas, manutenção de instalações higienizadas, monitoramento dos recursos humanos e das técnicas de produção e distribuição dos alimentos prontos [9].

Tendo em vista a importância do controle e estudo das condições higiênico - sanitárias dos serviços de alimentação para a saúde humana, objetivou-se investigar essas condições em UANs hospitalares da região oeste de Santa Catarina (SC) pela avaliação das não conformidades e adequação às diretrizes e as normas de Boas práticas de fabricação de acordo com a legislação vigente. Justifica-se ainda, pela UAN hospitalar ser um local que tem como finalidade favorecer a promoção do consumo de alimentos 
seguros, contribuindo com a qualidade de vida e recuperação da saúde dos pacientes.

\section{METODOLOGIA}

\section{Caracterização do local}

Este estudo foi realizado em quatro unidades de alimentação e nutrição hospitalares da região oeste de Santa Catarina, compreendendo os municípios de Seara, Quilombo, Abelardo Luz e Chapecó, referenciados como estabelecimentos A, B, C e D (atribuição aleatória), no período de janeiro a julho de 2012.

O hospital A localizado em Seara possui 51 leitos e aproximadamente realiza 1800 atendimentos/mês. O hospital B, situado no município de Quilombo, mantém 47 leitos e faz 1100 atendimentos/mês. $\mathrm{O}$ hospital $\mathrm{C}$ estabelecido em Abelardo Luz dispõe de 41 leitos e exerce 1000 atendimentos/mês e o hospital D (localizado em Chapecó) mantém 140 leitos e efetiva 3800 atendimentos/mês. Todos os hospitais possuem serviço centralizado e de autogestão.

\section{Coleta de dados}

A coleta de dados foi iniciada somente após a autorização dos Hospitais para a realização do estudo. Para a coleta dos dados a pesquisadora realizou visitas aos locais com objetivo de implementar a observação direta complementada por informações fornecidas pelo nutricionista ou colaboradores que trabalham no hospital. As visitas foram realizadas em distintos dias e horários, de acordo com a disponibilidade dos hospitais.

Como inexiste até o momento (processo de elaboração e tramites para a publicação do presente trabalho) uma legislação especifica com um check list para a por avaliar as mesmas por meio da adoção de um formulário em forma de lista de verificação, validado e publicado pela Vigilância Sanitária, baseado nas resoluções RDC no 216 de 15 de setembro de 2004 que dispõe sobre o regulamento técnico de Boas Práticas para serviços de alimentação, e a RDC no 275 de 21 de outubro de 2002 do Ministério da Saúde que dispõe sobre o regulamento técnico de Procedimentos Operacionais Padronizados aplicados aos estabelecimentos produtores/industrializadores de alimentos.
Por meio da lista de verificação foram analisados 108 itens distribuídos da seguinte forma:

- Edificações e instalações (n=37);

- Equipamentos, móveis e utensílios ( $\mathrm{n}=10)$;

- Controle integrado de vetores e pragas urbanas $(n=3)$;

- Abastecimento de água $(\mathrm{n}=6)$;

- Manejo de resíduos (n=4);

- Manipuladores ( $\mathrm{n}=9)$;

- Matérias primas, ingredientes e embalagens $(n=7)$;

- Preparação do alimento (n=16);

- Armazenamento e transporte do alimento preparado $(\mathrm{n}=3)$;

- Exposição ao consumo do alimento preparado (n=5);

- Documentação e registro $(\mathrm{n}=8)$;

Segundo a RDC no $275^{\text {[11] }}$ para a obtenção do resultado da avaliação das condições higiênico-sanitárias, a partir de um cálculo, são considerados os itens julgados e os itens atendidos. Para os itens com resposta C (conforme), foi atribuído o valor um (1) e para os itens cuja resposta foi $\mathrm{NC}$ (não conforme), foi atribuído o valor zero (0). As respostas C foram somadas e em seguida foi calculada a percentagem de adequação, por meio da equação:

$\mathrm{PA}=\frac{\text { Itens atendidos } \mathrm{x} 100}{\text { Itens julgados }}$

Onde:

$\mathrm{PA}=$ Percentagem de adequação

Itens atendidos $=$ número de respostas $\mathrm{C}$

Itens julgados $=$ número total de respostas $(\mathrm{C}$ e NC)

A partir desse cálculo, os estabelecimentos foram agrupados de acordo com o percentual dos itens atendidos em: 
Grupo 1: Bom (76 a 100\% de itens atendidos);

Grupo 2: Regular (51 a 75\% dos itens atendidos) e

Grupo 3: Ruim (0 a 50\% dos itens atendidos)

Vale ressaltar que além da avaliação global da unidade, cada item foi avaliado separadamente demonstrando as porcentagens de adequação de cada um.

Foi entregue para cada unidade de alimentação hospitalar uma relação das não conformidades encontradas na pesquisa para subsidiar a atuação da nutricionista responsável técnica do hospital, nas estratégias para adequação.

\section{RESULTADOS E DISCUSSÃO}

A análise dos dados obtidos a partir da lista de verificação possibilitou observar que nenhuma UAN hospitalar avaliada foi classificada no Grupo 3 - Ruim, considerado o de pior qualidade higiênico-sanitária. Duas UANs foram classificadas no Grupo 2 - Regular e duas no Grupo 1 - Bom.

As Unidades A e B foram classificadas no Grupo 2 (51 a $75 \%$ dos itens atendidos) atendendo 65\% e 68,2\% respectivamente. Já as UANs hospitalares C e D foram classificadas no Grupo 1 (76 a 100\% de itens atendidos) apresentando-se como "Bom" atendendo $87,5 \%$ e $98,13 \%$ dos itens atendidos.

Os resultados obtidos (valores médios) por meio da adoção da lista de verificação aplicada nas UANs hospitalares estão apresentados na Tabela 1.

Tabela 1. Resultados das médias obtidas com a lista de verificação aplicada nas UANs Hospitalares da região oeste de Santa Catarina.

\begin{tabular}{|c|c|c|c|c|}
\hline \multirow{3}{*}{ Itens avaliados } & \multicolumn{4}{|c|}{ UANs Hospitalares } \\
\hline & \multicolumn{4}{|c|}{$(\%)$} \\
\hline & A & B & $\mathrm{C}$ & $\mathrm{D}$ \\
\hline Edificações e instalações & 56,2 & 60,0 & 71,4 & 97,2 \\
\hline Equipamentos, móveis e utensílios & 50,0 & 70,0 & 100,0 & 100,0 \\
\hline Controle integrado de pragas & 100,0 & 100,0 & 100,0 & 100,0 \\
\hline Abastecimento de água & 100,0 & 100,0 & 100,0 & 100,0 \\
\hline Manejo de resíduos & 75,0 & 100,0 & 100,0 & 100,0 \\
\hline Manipuladores & 77,7 & 77,0 & 100,0 & 88,8 \\
\hline Matérias primas, ingredientes e embalagens & 71,4 & 85,7 & 85,7 & 100,0 \\
\hline Preparação do alimento & 68,7 & 68,7 & 93,7 & 100,0 \\
\hline Armazenamento e transporte & 33,3 & 66,6 & 66,6 & 100,0 \\
\hline Exposição consumo do alimento preparado & 100,0 & 100,0 & 100,0 & 100,0 \\
\hline Documentação e registro & 0,00 & 14,28 & 100,0 & 100,0 \\
\hline
\end{tabular}

Como foi possível observar a UAN hospitalar $\mathrm{D}$ foi a que apresentou UANs, enquadrando-se no Grupo 1 (valores médios em conformidade).

Verificou-se que a UAN A, foi classificada no geral no Grupo 2 - Regular (51 a 75\% de adequação), porém em relação a percentagem de adequação de alguns itens avaliados como "Documentação e registro" (0\%) "Armazenamento e transporte" (33,3\%) e "Equipamentos, móveis e utensilios" (50\%) permitem o enquadramento no Grupo 3 - Ruim (0 a $50 \%$ dos itens atendidos).

Farias et al. [12] avaliaram as Boas práticas de uma UAN hospitalar em dois períodos diferentes, e o item "equipamentos, móveis e utensílios" foi o que apresentou a menor percentagem de conformidades quanto às boas práticas $(19,04 \%)$ no primeiro período 
de avaliação, apresentando resultados semelhantes aos encontrados por meio da presente pesquisa.

A UAN hospitalar B também apresentou o item "Documentação e registro" com percentagem $(14,28 \%)$ também dentro do Grupo 3. O mesmo acontece com a UAN hospitalar C, que foi classificada no geral no Grupo 1 - Bom (76 a 100\% dos itens atendidos), porém nos itens "Armazenamento e transporte" (66,6\%) e "Edificações e instalações" $(71,4 \%)$ permitem o enquadramento no grupo 2 Regular (51 a $75 \%$ dos itens atendidos).

Ao avaliar as edificações e instalações de dois hospitais do município de Castanhal-Pará, Silva e Silva [13] verificaram irregularidades distintas daquelas encontradas neste trabalho, com média de $21 \%$ de conformidade com as BPF.

Ainda no estudo de Farias et al., [12] foi constatado que em nenhum dos dois períodos avaliados, a unidade de alimentação do hospital foi classificada no Grupo 1 - Bom (76 a 100\% dos itens atendidos). $\mathrm{O}$ único item que se enquadrou no Grupo 2 - Regular (51 a $75 \%$ dos itens atendidos), foi o de "manipuladores" no segundo período de avaliação, ficando os demais itens classificados no Grupo 3 Ruim (0 a $50 \%$ dos itens atendidos), diferentemente dos resultados encontrados neste estudo.

Em relação às edificações e instalações, podese verificar que nenhuma UAN hospitalar possuía as portas da área de preparação e armazenamento de alimentos dotadas de fechamento automático, o que facilita a entrada de pragas e vetores. Em contrapartida, todas apresentavam telas milimétricas nas janelas. Segundo a RDC ㄲo $275^{[11]}$ as portas externas devem ter fechamento automático (mola, sistema eletrônico ou outro) e com barreiras adequadas para impedir a entrada de vetores e outros animais (telas milimétricas ou outro sistema).

Apenas a UAN D possuía edificações e instalações projetadas de forma a possibilitar um fluxo ordenado e sem cruzamento de forma a facilitar a manutenção e a limpeza. Para Akutsu et al., ${ }^{[1]}$ o layout das UANs bem como o seu processo de manipulação deve seguir um fluxo higiênico adequado e ininterrupto. A área de alimentos crus deve estar separada da área dos alimentos preparados e prontos para consumo, minimizando, assim, o risco de contaminação.
As Unidades A e B apresentaram inconformidades em relação ao piso, parede e teto, onde os mesmos encontravam-se fora da legislação sem revestimento liso, impermeável e lavável. Tanto o piso, quanto o teto e paredes destas unidades apresentavam pontos de umidade e rachaduras. No estudo de Guedes [14] o autor observou que $75 \%$ das unidades de alimentação de hospitais da rede pública de Brasilia (DF), apresentavam inconformidade nos pisos, paredes, tetos, portas e janelas.

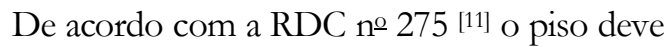
ser de material que permite fácil e apropriada higienização. $\mathrm{O}$ teto em acabamento liso, em cor clara, impermeável, de fácil limpeza e, quando for o caso, desinfecção, Em adequado estado de conservação (livre de trincas, rachaduras, umidade, bolor, descascamentos e outros). As paredes em acabamento liso, impermeável, de fácil higienização até uma altura adequada para todas as operações e de cor clara.

Em relação à iluminação, a Unidade $\mathrm{D}$ foi a única que apresentou luminárias protegidas contra explosão ou queda acidental localizadas sobre a área de preparação dos alimentos. Segundo a RDC no $275^{[1]]}$ as luminárias com proteção adequada contra quebras e em adequado estado de conservação.

Das quatro unidades, três apresentavam lavatórios exclusivos para a higienização das mãos exceto a unidade A. Sendo que a Unidade B não apresentava o lavatório em posição estratégica em relação ao fluxo de preparo dos alimentos e a Unidade C não possuía lixeiras com tampa com acionamento por pedal, tornando-se assim, questionável sua real eficiência.

Em relação aos equipamentos, móveis e utensilios, as unidades $\mathrm{A}$ e $\mathrm{B}$ não possuíam nenhum tipo de registro de manutenção e calibração dos equipamentos e limpeza. Sendo assim dificulta o conhecimento sobre a frequência, modo ou eficácia dos procedimentos de higienização. Além disso, não realizavam o controle de diluição, tempo de contato e aplicação dos saneantes durante o procedimento de higienização dos equipamentos. A unidade A apresentava a superfície de alguns equipamentos e móveis com rugosidades e frestas facilitando a contaminação.

Valejo et al. [15], quando vistoriaram 52 serviços de alimentação, na cidade de Presidente Prudente - SP, 
constataram que os maiores problemas foram em relação à má conservação de utensílios e equipamentos.

As superfícies e equipamentos que entram em contato com o alimento durante a sua preparação podem se tornar focos de contaminação, principalmente quando não forem bem higienizados, $\mathrm{o}$ que sugere a necessidade de medidas efetivas de controle [16].

No quesito controle integrado de vetores e pragas urbanas, observou-se que todas as unidades estavam em conformidade com a legislação vigente. A totalidade das unidades realizavam controle químico por meio de contratação de empresa especializada e todas apresentaram os laudos. Para a RDC no 275 [11] deve-se haver ausência de vetores e pragas urbanas ou qualquer evidência de sua presença como fezes, ninhos e outros. A adoção de medidas preventivas e corretivas com o objetivo de impedir a atração, o abrigo, o acesso e ou proliferação de vetores e pragas urbanas. Em caso de adoção de controle químico é preciso a existência de comprovante de execução do serviço expedido por empresa especializada.

Em estudo realizado por Cruz et al., [17] os autores constataram que o controle de pragas em uma unidade produtora de alimentos mostrava-se totalmente inexistente, podendo realmente acarretar prejuízos para a segurança microbiológica do produto, sendo os dados encontrados por estes autores opostos aos encontrados no presente estudo. A RDC no $216^{[18]}$ estabelece que devem ser implantados procedimentos eficazes e contínuos visando a prevenção ou minimização da presença de vetores e pragas urbanas.

Em relação ao abastecimento de água, todas as Unidades apresentavam água potável, sendo a potabilidade atestada semestralmente e trimestralmente. $\mathrm{O}$ controle da água era realizado por outros setores do hospital, porém todas possuíam laudos e registros. No estudo citado por Cruz et al. [17], os autores registram que a qualidade da água é de fundamental importância, já que a mesma é considerada um veículo para muitos micro-organismos patogênicos.

Quanto ao manejo de resíduos, somente a Unidade A não possuía recipientes identificados e íntegros e em número e capacidade suficientes para conter os resíduos. Todas as unidades apresentavam uma área especifica para o armazenamento do lixo, recipientes dotados de tampa sem contato manual, os resíduos eram coletados freqüentemente evitando acúmulos e eram estocados em local isolado da área de preparação e armazenamento dos alimentos.

Oliveira et al. ${ }^{[19]}$ relatam que nem sempre as não conformidades estão relacionadas à falta de recursos financeiros e sim associadas à ausência de informação/conhecimento dos responsáveis pela organização, gerenciamento e operacionalização das atividades. O investimento financeiro na adequação do manejo correto de lixo é pequeno em relação ao custo/benefício e pode ser facilmente adequado na UAN onde as não conformidades foram encontradas.

Em se tratando dos manipuladores, todos apresentavam asseio pessoal, boa apresentação, com uniformes de trabalho completos e limpos, com todos os equipamentos de proteção individual (EPI), cabelos presos, sem adornos e maquiagem. A lavagem correta e periódica das mãos era realizada nas quatro unidades. Estudo citado por Ribeiro e Schmidt ${ }^{[20]}$ registra que a contaminação cruzada pode ocorrer através das próprias mãos dos manipuladores.

As Unidades A e B, não apresentam registros de capacitação e treinamentos periódicos para os manipuladores, vale ressaltar que ambas as unidades não possuíam responsável técnico (nutricionista) no local. A equipe dessas unidades era comandada por enfermeiras e segundo elas uma nutricionista prestava serviços para o hospital organizando as dietas hospitalares. Durante a rotina na cozinha, ninguém acompanhava o controle higiênico sanitário e a produção. A presença do responsável técnico parece ser um importante recurso para as distinções entre as UANs.

Para a garantia da qualidade sanitária das refeições servidas, os estabelecimentos produtores de refeição devem apresentar um Responsável Técnico (RT) pelas atividades de manipulação dos alimentos [21].

A capacitação dos manipuladores de alimentos por meio de treinamentos significa contribuir não apenas para a melhoria da qualidade higiênico-sanitária, mas para o aperfeiçoamento das técnicas e processamento utilizados [22]. Os perigos microbiológicos são as principais causas de contaminação dos alimentos e os manipuladores constituem a origem do problema e são grandes responsáveis pela sua contaminação microbiológica [23]. 
De acordo com a RDC no $216^{[18]}$ deve haver programa de capacitação adequado e contínuo relacionado à higiene pessoal e à manipulação dos alimentos e existência de registros dessas capacitações. A capacitação deve ser comprovada mediante documentação.

A UAN hospitalar $\mathrm{C}$ foi a única que apresentou cartazes de orientação aos manipuladores sobre a correta lavagem e anti-sepsia das mãos afixados em locais visíveis inclusive nas instalações sanitárias e lavatórios.

De acordo com as matérias-primas, ingredientes e embalagens, das 4 unidades, duas delas (A e B) não apresentavam registro de inspeção das matérias primas e ingredientes durante a recepção. Devem ser estabelecidos padrões de identidade e qualidade para matérias primas ingredientes $\mathrm{e}$ embalagens. Os mesmos devem ser devolvidos na medida em que esse monitoramento indique um desvio que possa comprometer a qualidade do produto final. O monitoramento deve ser registrado em planilhas servindo de certificação para os fornecedores. Estudo realizado por Cardoso et al. [24] mostrou que entre os responsáveis pelo recebimento nos estabelecimentos, $100 \%$ tinham o hábito de verificar a validade dos alimentos, 85\% observavam aspectos sensoriais do produto e $95 \%$ preocupavam-se em avaliar as condições de embalagem. Os números encontrados na presente pesquisa são discordantes do referido estudo. A primeira etapa de controle higiênico sanitária no estabelecimento é o recebimento dos alimentos e deve englobar atividades de conferência dos produtos recebidos.

A Unidade C não armazenava seus ingredientes e matérias-primas de forma correta nas prateleiras, sendo que as mesmas não estavam afastadas da parede. Segundo a RDC no $275^{\text {[11] todos os }}$ alimentos devem ser armazenados e separados por tipo ou grupo, sobre estrados distantes do piso, ou sobre paletes, bem conservados e limpos, afastados das paredes e distantes do teto, de forma a permitir apropriada higienização, iluminação e circulação de ar.

Durante a preparação do alimento, as Unidades A, B e C não adotavam medidas a fim de minimizar os riscos de contaminação cruzada havendo contato direto ou indireto entre alimentos crus, semipreparados e prontos para o consumo, justamente por não terem as áreas separadas por meios físicos. Apesar de a Unidade $\mathrm{C}$ ter as áreas separadas, na hora da preparação não é verificado este tipo de cuidado. Nas unidades $\mathrm{A}$ e B não era verificada a temperatura durante a cocção ou durante a distribuição e o tempo de exposição. A relação binômio tempo e temperatura é fundamental para a manutenção da qualidade dos produtos alimentícios ${ }^{[25]}$.

Luchese et al. [26] destacam a necessidade de se estabelecer controle e monitoramento de tempo e temperatura nas etapas de armazenamento, descongelamento, pré-preparo, preparo, resfriamento, reaquecimento, adição de ingredientes, espera póscocção, distribuição e utilização de sobras em UANs.

A respeito do armazenamento e transporte do alimento preparado, as unidades $\mathrm{C}$ e $\mathrm{A}$ não possuíam os carrinhos de transporte fechados.

No quesito documentação e registro, foi observado que somente as Unidades C e D possuíam o Manual de Boas praticas e procedimentos Operacionais Padronizados.

De acordo com a Tabela 2 pode-se verificar as principais não-conformidades encontradas nas UANs integrantes da pesquisa. 
Tabela 2. Principais não conformidades encontradas nas UANs Hospitalares da Região Oeste de Santa Catarina.

\begin{tabular}{|c|c|c|}
\hline Categorias & Não conformidades & Percentual \\
\hline \multirow{5}{*}{ Edificações e instalações } & $\begin{array}{l}\text { Edificações e instalações mal projetadas, com fluxo desordenado e com } \\
\text { cruzamentos }\end{array}$ & 75 \\
\hline & Áreas sem separação por meios físicos ou técnicos & 50 \\
\hline & Piso, paredes e tetos fora dos padrões sem revestimento liso, impermeável e lavável & 75 \\
\hline & Portas sem fechamento automático e não ajustadas aos batentes & 75 \\
\hline & Luminárias sem proteção contra explosão & 50 \\
\hline \multirow{3}{*}{$\begin{array}{l}\text { Equipamentos, móveis e } \\
\text { utensílios }\end{array}$} & Equipamentos em ruim estado de conservação & 25 \\
\hline & Sem registro de manutenção e calibração & 50 \\
\hline & Sem registro de limpeza & 50 \\
\hline Manejo de resíduos & Recipientes sem identificação & 25 \\
\hline \multirow[b]{2}{*}{ Manipuladores } & Sem cartazes de orientação da correta lavagem de mãos & 75 \\
\hline & $\begin{array}{l}\text { Ausência de capacitação e treinamentos periódicos para os manipuladores } \\
\text { devidamente registrados }\end{array}$ & 50 \\
\hline \multirow{3}{*}{$\begin{array}{l}\text { Matérias-primas, } \\
\text { ingredientes e embalagens }\end{array}$} & Sem critérios para avaliação e seleção de fornecedores & 25 \\
\hline & Registro de inspeção das matérias primas e ingredientes na recepção & 50 \\
\hline & Armazenamento sobre palletes ou prateleiras de forma errada & 25 \\
\hline \multirow{3}{*}{ Preparação do alimento } & $\begin{array}{l}\text { Preparação do alimento errada, com contato direto entre alimentos crus, semi- } \\
\text { preparados e prontos }\end{array}$ & 75 \\
\hline & Não há verificação da temperatura e do tempo utilizado & 50 \\
\hline & Temperaturas dos equipamentos frios não são monitoradas e registradas & 50 \\
\hline \multirow[b]{2}{*}{$\begin{array}{l}\text { Armazenamento e } \\
\text { transporte do alimento }\end{array}$} & Não há controle de temperatura na distribuição do alimento & 50 \\
\hline & $\begin{array}{l}\text { Os meios de transporte do alimento preparado não possuem cobertura para } \\
\text { proteção de carga }\end{array}$ & 50 \\
\hline \multirow{3}{*}{ Documentação e registro } & Não possui o Manual de Boas Práticas & 50 \\
\hline & Não possui POP`s & 50 \\
\hline & $\begin{array}{l}\text { Responsáveis pelas atividades e manipuladores não possuem comprovantes de } \\
\text { capacitação }\end{array}$ & 50 \\
\hline
\end{tabular}

\section{CONCLUSÃO}

A partir da análise dos resultados obtidos por meio da adoção da lista de verificação pode-se concluir que as quatro UANs hospitalares apresentaram um bom percentual de adequação e nenhuma se encontrou na classificação Ruim.

Observou-se que as duas UANs que se classificaram no Grupo 2 - Regular, são as Unidades que não possuem atuação no local. As outras duas UANs possuem nutricionista que atuam como responsável técnico. Portanto, é provável que seja possivel atribuir a diferença observada nos locais ao efetivo trabalho do nutricionista responsável.

A UAN D, com o melhor percentual possui nutricionista com carga horária de 40 horas semanais. A UAN C que também foi classificada no Grupo 1 - 
Bom, e obteve o segundo melhor percentual, possui nutricionista, mas não em período integral.

Nesse sentido, é provável que a presença do nutricionista responsável técnico faça com que as unidades, integrantes da pesquisa, sejam atentas para o cumprimento da maioria dos itens do checklist. No entanto, as UANs com responsável técnico também apresentaram falhas, em vista disso espera-se a superação dos desvios apontados por meio da utilização do instrumento, em especial dos itens que estariam vinculados à garantia da qualidade higiênico-sanitária dos alimentos, evitando as DTA`s e o comprometimento da recuperação dos pacientes internados nos hospitais avaliados.

\section{REFERÊNCIAS}

[1] Akutsu RC, Botelho RA, Camargo EB, Sávio KLO, Araújo WC. Adequação das boas práticas de fabricação em serviços de alimentação. Rev. Nutr. 2005;18(3):419-27.

[2] Ministério da Saúde (BR). Estudo de caso Brasil: a integração das ações de alimentação e nutrição nos planos de desenvolvimento nacional para o alcance das metas do milênio no contexto do direito humano a alimentação adequada. Brasília: Ministério da Saúde; 2005.

[3] Organização Mundial de Saúde. Foodborne disease [internet]. Genebra; 2010 [acesso em 03 jun 2010]. Disponível em: hhtp: www.who.int

[4] Genta TMS, Mauricio AA, Matioli G. Avaliação das boas práticas através de "check-list" aplicado em restaurantes selfservice da região central de Maringá, estado do Paraná. Acta Sci Health Sci. 2005;27(2):151-156.

[5] Alves MG, Ueno M. Restaurantes self-service: segurança e qualidade sanitária dos alimentos servidos. Rev. Nutr. 2010;23(4):573-80.

[6] Schneider AP. Fornecimento de hortifrutigranjeiros para unidades de alimentação e nutrição hospitalares. Ciênc. Tecnol. Aliment. 2006;26(2):253-58.

[7] Martinelli C. Avaliação microbiológica de produtos cárneos distribuídos aos pacientes em um hospital particular de Volta Redonda - RJ [dissertação]. Rio de Janeiro: Universidade Federal Rural do Rio de Janeiro; 2007.

[8] Souza AA; Proença RPC. Tecnologias de gestão dos cuidados nutricionais: recomendações para qualificação do atendimento nas unidades de alimentação e nutrição hospitalares. Rev. Nutr. 2004,17(4):425-436.
[9] Sousa CL, Neves, ECA, Lourenço LFH, Costa EB, Monteiro RRC. Diagnóstico das condições higiênicosanitárias e microbiológicas de empresas fornecedoras de comidas congeladas light na cidade de Belém/PA. Alim. Nutr. 2009;20(3):375-81.

[10] Badaró AC, Azeredo RM, Almeida ME. Vigilância Sanitária de Alimentos: uma revisão. Revista Digital de Nutrição: Nutrir Gerais. 2007,1(1):1-25.

[11] Ministério da Saúde (BR). RDC no 275, de 21 de outubro de 2002. Dispõe sobre o regulamento técnico de procedimentos operacionais padronizados aplicados aos estabelecimentos produtores/industrializadores de alimentos. Diário Oficial da União. 06 nov 2002; Seção 1:126.

[12] Farias JKR, Pereira MMS, Figueiredo EL. Avaliação de boas práticas e contagem microbiológica das refeições de uma unidade de alimentação hospitalar, do município de São Miguel do Guamá - Pará. Alim. Nutr. 2011;22(1):113-19.

[13] Silva RF, Silva SF. Avaliação das condições higiênicosanitárias e implantação de boas práticas de fabricação em cozinhas hospitalares da cidade de Castanhal - Pará. Belém: Universidade do Estado do Pará; 2005. Trabalho de Conclusão de Curso.

[14] Guedes T. Avaliação das condições higiênico sanitárias das cozinhas hospitalares de Asa Sul do Distrito Federal. Brasilia: Universidade de Brasília; 2009. Trabalho de Conclusão de Curso.

[15] Valejo FAM, Andrés CR, Mantovan FB, Rister GP, Santos GD, Andrade FF. Vigilância sanitária: avaliação e controle de qualidade dos alimentos. Rev. Hig. Alim. 2003;17(106):16-21.

[16] Silva Júnior EA. Manual de controle higiênico-sanitário em serviços de alimentação. 6. ed. São Paulo: Varela, 2007.

[17] Cruz AG, Cenci AS, Maia MCA. Pré- requisitos para implementação do sistema APPCC em uma linha de alface minimamente processada. Ciênc. Tecnol. Aliment. 2006;26(1):104-09.

[18] Ministério da Saúde (BR). RDC no 216, de 15 de setembro de 2004. Dispõe sobre o regulamento técnico de Boas Práticas para serviços de alimentação. Diário Oficial da União. 16 set 2004.

[19] Oliveira MN, Brasil ALD, Taddei JAAC. Avaliação das condições higiênico-sanitárias das cozinhas de creches públicas e filantrópicas. Ciência \& Saúde Coletiva. 2008;13(3):1051-1060. 
[20] Ribeiro KL, Schmidt V. Caracterização de manipuladores de alimentos em escolas municipais de Viamão, RS. Rev. Hig. Alim. 2007,21(157):58-64.

[21] Barros APN, Costa TF, Pereira SCL, Brito MJM, Monteiro MAM, Abreu MNS. Perfil sociodemográfico, de conhecimentos administrativos, em legislação sanitária de gerentes e segurança alimentar em unidades produtoras de refeições comerciais. Nutrire. 2011;36(2):61-76.

[22] Saccol ALF, Rubim BA, Mesquita MO, Welter L. Importância de treinamentos de manipuladores em Boas Praticas. Disc. Scientia. Série: Ciências da Saúde. 2006;7(1): 91-99.

[23] Souza LHL A manipulação inadequada dos alimentos: fator de contaminação. Rev. Hig. Alim. 2006;20(146):32-39.
[24] Cardoso RCV, Souza EVA, Santos PQ. Unidades de alimentação e nutrição nos campi da Universidade Federal da Bahia: um estudo sob a perspectiva do alimento seguro. Rev. Nutr. 2005;18(5):669-680.

[25] Silva Neto M. Diagnóstico situacional da utilização das ferramentas de segurança na produção de alimentos das cozinhas das Unidades de Alimentação e Nutrição dos Hospitais de Brasília - DF [dissertação]. Brasília: Universidade de Brasília; 2006.

[26] Luchese RH, Borges JTS, Maia LH, Freitas AS. Análises de perigos e pontos críticos de controle na preparação da carne bovina assada em unidades de alimentação e nutrição. Rev. Hig. Alim. 2004;17(108):36-41. 